\title{
PERCEPCIONES SOBRE ALGUNAS CONDUCTAS AMBIENTALES COTIDIANAS DE LA POBLACIÓN COSTARRICENSE
}

\section{PERCEPTIONS ABOUT SOME DAILY ENVIRONMENTAL BEHAVIOUR OF THE COSTA RICAN POPULATION}

\author{
Martín Solís Salazar*
}

RESUMEN

Este estudio busca aportar elementos que permitan describir la conducta proambiental de la población costarricense. La información utilizada proviene de una encuesta telefónica realizada a la población costarricense mayor de 17 años, en total se aplicaron 1200 entrevistas. Se analiza el accionar ambiental en acciones cotidianas, la denuncia de situaciones que afectan el ambiente y la participación de campañas ambientales. Además, se evalúa la relación entre las características sociodemográficas de la población y la conducta ambiental.

PALABRAS CLAVE: COSTA RICA * CONDUCTA AMBIENTAL * MEDIO AMBIENTE * POBLACIÓN * CULTURA AMBIENTAL * CIENCIAS AMBIENTALES

\section{ABSTRACT}

The research seeks to find elements to describe the environmental behaviour of Costa Rican population. To pick up information, was applied a telephonic survey to people older than 17 years old, 1200 interviews were collected. Environmental action was analyzed in daily activities, reporting of situations that affect the environment and the participation of environmental campaigns. Besides, were analyzed the relation between demographics features and environmental behaviour.

KEYWORDS: COSTA RICA * ENVIRONMENT BEHAVIOUR * ENVIRONMENT * POPULATION * ENVIRONMENTAL CULTURE * ENVIRONMENTAL SCIENCES

Instituto de Estudios Sociales en Población de la Universidad Nacional de Costa Rica.

martin12cr@yahoo.com 


\section{INTRODUCCIÓN}

"El mundo vive hoy en día una crisis ecológica sin precedentes donde se explotan los recursos naturales sin tener en cuenta las necesidades futuras" (Hassan, 2005). Todo ello acrecentado por el modelo capitalista que presiona a las sociedades en función del consumo exacerbado y la explotación desmedida de recursos. Dentro de este modelo, el ambiente es considerado como una forma de capital y los ciclos ecológicos se toman como servicios que pueden ser ingresados al mercado (Gudynas, 2002: 31).

A nivel mundial, se ha desencadenado una alarma ambiental, que aboga por la protección y la conservación de los recursos naturales, por lograr una integridad ecológica (Tucker, 2005: 25 y Mackey, 2005: 67). La carta de la tierra es enfática en que el ser humano debe optar por "una sociedad global para cuidar la tierra o arriesgar a la destrucción de nosotros mismos y la diversidad de vida" (Corcoran et ál., 2005: 24).

Ante tal contexto, cabe preguntar: ¿cuál será el compromiso cotidiano de la sociedad costarricense con el ambiente?, ¿ejerce la población costarricense una conducta ambiental en su diario vivir? En este estudio, se entiende el término "conducta ambiental" como aquellas acciones orientadas a la protección y conservación del ambiente (Corral, 1998).

Estudios realizados por el Instituto de Estudios Sociales en Población (IDESPO) de la Universidad Nacional durante los años 2005, 2006 y 2007, dan puntilladas de respuesta a las preguntas. Por ejemplo: en ellos se encuentra que la participación de la población en grupos que trabajan por el ambiente es un poco menor al 30\% de la población, no obstante, se detalla que en otras conductas ambientales el compromiso es más extenso, como es el caso del ahorro de agua (Bustos et ál., 2005: 6-13; Bustos et ál., 2006: 12-21; Sandoval et ál., 2007). También se muestran ciertas actitudes proambientales que en última instancia podrían promover la conservación y la protección del ambiente. Por otra parte, Lentini (2005: 16) analiza los resultados de una encuesta realizada en el año 2002, en donde se establece que el $60 \%$ de la población costarricense actúa parcialmente en favor del ambiente.
Ahora bien, estos estudios no tenían como orientación principal dar respuestas a las preguntas planteadas con anterioridad y preservan la necesidad de describir aún más la conducta ambiental cotidiana de la población del costarricense, además de monitorearla a través del tiempo.

El objetivo de esta investigación es aportar elementos que contribuyan a describir la conducta ambiental cotidiana de la población costarricense y por esa línea, comprender aun más el compromiso de la población hacia la protección y conservación del ambiente. Para cumplir con el objetivo, se realizó una encuesta de opinión en la que se capturaron las percepciones de la población sobre su conducta ambiental.

Como parte de la descripción, se pretende evaluar los cambios en la conducta ambiental a través de las principales variables sociodemográficas de la población. Varias investigaciones han resaltado la influencia de las características sociodemográficas sobre la conducta ambiental, por ende, se han considerado como factor predictivo y diferenciador (Barr y Gilg, 2007: 370; González, 2002: 140; Berger, 1997: 515; Granzin y Olsen, 1991:1; Hines et ál., 1986: 7).

Es importante destacar que son muchas las acciones ambientales que pueden describir la conducta ambiental cotidiana de la población. Por esta razón, se enfatiza solamente en algunas acciones propias de la cotidianidad en las que todas las personas están envueltas, como lo son: ahorro de electricidad, ahorro de agua, separación de desechos sólidos y hábitos de compra. También se evalúa el accionar de la población hacia la participación en campañas de beneficio ambiental y el accionar hacia la denuncia de situaciones que afectan el ambiente.

\section{DATOS Y MÉTODOS}

\section{FUENTES DE DATOS}

Los datos provienen de una encuesta telefónica realizada en el Instituto de Estudios Sociales en Población (IDESPO) de la Universidad Nacional de Costa Rica, entre el 17 y el 30 de mayo del año 2010. A continuación se describen las principales características. 


\section{POBLACIÓN DE ESTUDIO}

Todo(a) costarricense o extranjero(a) con dos o más años de residir en el país, mayor de edad y residente en viviendas particulares que poseen teléfono residencial.

\section{DISEÑO MUESTRAL}

La encuesta fue telefónica y se aplicó a 1200 personas. Este tamaño de muestra se calculó buscando obtener un error máximo de 2,8 puntos porcentuales con un $95 \%$ de confianza en la mayor parte de las variables en estudio.

El tamaño de muestra se distribuyó por sexo y grupos de edad, para cumplir con una cuota probabilística que replicara la distribución de la población en esas características particulares, como se presenta en la tabla 1.

TABLA 1

DISTRIBUCIÓN PORCENTUAL DE LA POBLACIÓN POR SEXO Y EDAD

\begin{tabular}{lc}
\hline CARACTERÍSTICAS & $\%$ \\
\hline Sexo & \\
\hline Hombre & 47,5 \\
Mujer & 52,5 \\
\hline TOTAL & 100,0 \\
\hline Edad & \\
\hline De 18 a 24 años & 19,3 \\
De 25 a 34 años & 19,0 \\
De 35 a 44 años & 21,0 \\
De 45 a 54 años & 19,0 \\
De 55 a más años & 21,7 \\
\hline TOTAL & 100,0 \\
\hline
\end{tabular}

Fuente: Elaboración propia.

\section{TRABAJO DE CAMPO}

La selección de los números telefónicos se realizó de forma aleatoria a través de una base de datos, la cual contenía todos los números telefónicos de las viviendas particulares del territorio nacional.

\section{VARIABLES DE ANÁLISIS}

Con el fin de medir el accionar ambiental en ahorro de agua, ahorro de electricidad, separación de desechos y hábitos de compra, se establecieron ítems con una escala de respuesta Likert que varía de 1 a 5 , donde 5 significa que siempre se realiza la acción que establece el ítem, 4 casi siempre, 3 algunas veces, 2 casi nunca y 1 nunca. Las acciones de denuncia ambiental y participación en campañas de beneficio ambiental se miden a través de un único ítem.

Por su parte, las características sociodemográficas que se consideran en el estudio son: sexo, edad, nivel educativo, urbanismo del distrito de residencia y nivel socioeconómico del hogar de residencia. La variable urbanismo muestra el porcentaje de hogares que residen en la zona urbana en el distrito de residencia de la persona entrevistada. El nivel socioeconómico está representado por un indicador de tenencia de artefactos del hogar, que se construyó utilizando seis variables asociadas con la tenencia de artefactos en el hogar. A cada una de esas variables, se le asignó un peso, con el fin de que los artefactos que más se tienden a poseer, ejerzan menor influencia en el indicador. En la tabla 2 se muestran los artefactos con sus respectivos pesos.

TABLA 2

ARTEFACTOS SEGÚN PESO ASIGNADO

\begin{tabular}{lc}
\hline ARTEFACTOS & PESO \\
\hline Microondas & 1,0 \\
Ducha para agua caliente & 1,5 \\
Computadora & 1,5 \\
DVD (no computadora) & 1,5 \\
Celular & 1,0 \\
Carro (no de trabajo) & 2,0 \\
Conexión internet & 2,0 \\
\hline
\end{tabular}

Fuente: Elaboración propia.

Una vez asignados los pesos, se multiplicó el peso de cada artefacto por 1 en caso de que el hogar tuviese el artefacto y 0 en caso de que no lo tuviese. Por último, se construyó el 
indicador de pertenencias sumando los resultados de las multiplicaciones y transformándolo para que varíe de 0 a 10 , donde 10 representa el mayor nivel socioeconómico.

\section{TÉCNICAS DE ANÁLISIS}

La relación entre las características sociodemográficas y la escala de acciones cotidianas se evalúa a través de un modelo de regresión lineal por Mínimos Cuadrados Ordinarios, mientras que las relaciones entre las características sociodemográficas y las otras variables de conducta ambiental se analizan mediante modelos de regresión logística.

\section{RESULTADOS}

Se encuentra un mayor compromiso hacia las acciones de ahorro de electricidad, el cual es bastante amplio considerando que alrededor del $80 \%$ de las personas entrevistadas apagan siempre las luces encendidas y que en un $87 \%$ no deja los electrodomésticos encendidos sin usar (ver cuadro 1). La acción de desconectar los aparatos enchufados cuando se han dejado de usar, es la que presenta menor compromiso entre la acciones de ahorro de electricidad.

Las acciones de ahorro de agua se ubican en un segundo plano, las acciones de separación de desechos en un tercer plano y por último, aparecen las acciones relacionadas con hábitos de compra.

Las acciones anteriores conforman la escala de comportamiento proambiental en acciones cotidianas. La escala presenta un coeficiente Alfa de Cronbach de 0,70, lo cual indica que la escala tiene un nivel de confiabilidad aceptable.

CUADRO 1

PORCENTAJE DE PERSONAS QUE REALIZAN ACCIONES COTIDIANAS PROAMBIENTALES, SEGÚN PERCEPCIONES DE LA POBLACIÓN ENTREVISTADA 2010

\section{ACCIONES}

Apagar luces encendidas cuando nadie las está usando

Apagar la luz en la noche cuando sale de alguna habitación de la casa

Cerrar el tubo mientras se cepilla los dientes

Cerrar el tubo del baño mientras se enjabona

Cerrar el tubo mientras se enjabona las manos

Separar botellas plásticas del resto de la basura

Separar el vidrio del resto de la basura

Separar el papel del resto de la basura

Separar lata del resto de la basura

Llevar bolso a la pulpería o supermercado para evitar usar bolsas plásticas

Comprar jabones amigables con el ambiente
\% QUE SIEMPRE REALIZA LAS ACCIONES
79,2

79,2

62,6

55,3

53,8

51,7

44,5

44,4

31,3

25,5

ACCIONES

\% QUE NUNCA REALIZA LAS ACCIONES

Dejar electrodomésticos enchufados después de usarlos

Dejar electrodomésticos encendidos sin usar 13,5

Fuente: Elaboración propia. 
El puntaje de la escala varía de 0 a 100 , donde cero indica que no se realiza ninguna de las acciones ambientales y 100 que siempre se realizan todas las acciones anteriores. La escala presentó un puntaje de 64,4 con una desviación estándar de 17,5 (ver gráfico 1).

\section{GRÁFICO 1}

HISTOGRAMA DE LA ESCALA DE CONDUCTA AMBIENTAL EN ACCIONES COTIDIANAS

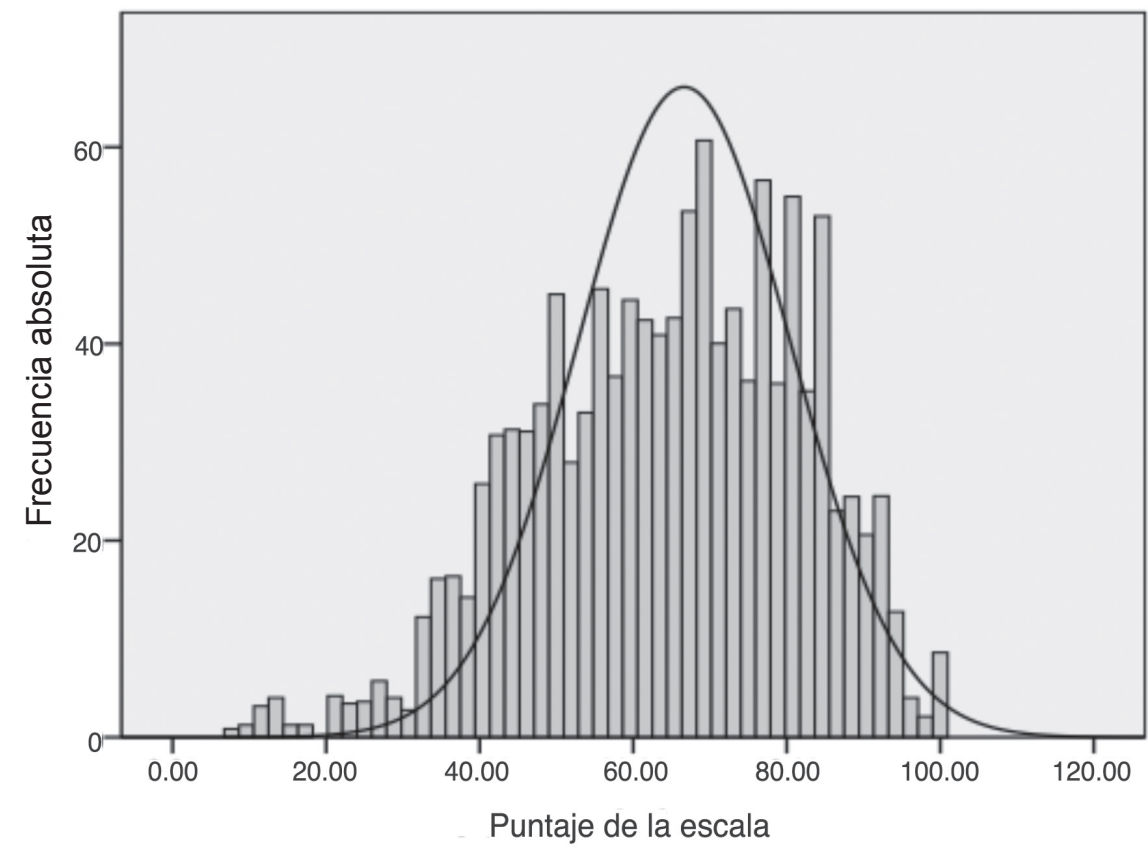

Fuente: Elaboración propia.

El puntaje obtenido da indicios de que en términos globales, la población no presenta un comportamiento predominantemente antiambiental en acciones cotidianas, pero tampoco altamente proambiental. Se podría considerar un comportamiento predominantemente proambiental cuando el puntaje supera los 75 puntos, ya que un individuo que realice casi siempre todas las acciones tendría un puntaje de 75 o bien, podría puntuar 75 indicando que realiza algunas de las acciones con menor frecuencia, pero tuvo que haber indicado total compromiso en otras acciones. Considerando 75 como el umbral, resulta importante destacar que tan solo un tercio de la población iguala o supera ese parámetro, es decir, solo un tercio muestra una conducta predominantemente proambiental en acciones cotidianas.

En algunos de los ítems que conforman la escala, se preguntaron las razones por las que no se realiza siempre la acción. En el cuadro 2 , se muestra la clasificación de las respuestas brindadas. La ausencia de costumbre es la justificación más frecuente que brindan las personas en casi todas las acciones.

1 En esta categoría se agruparon todas las respuestas relacionadas con olvido, descuido y falta de costumbre. 


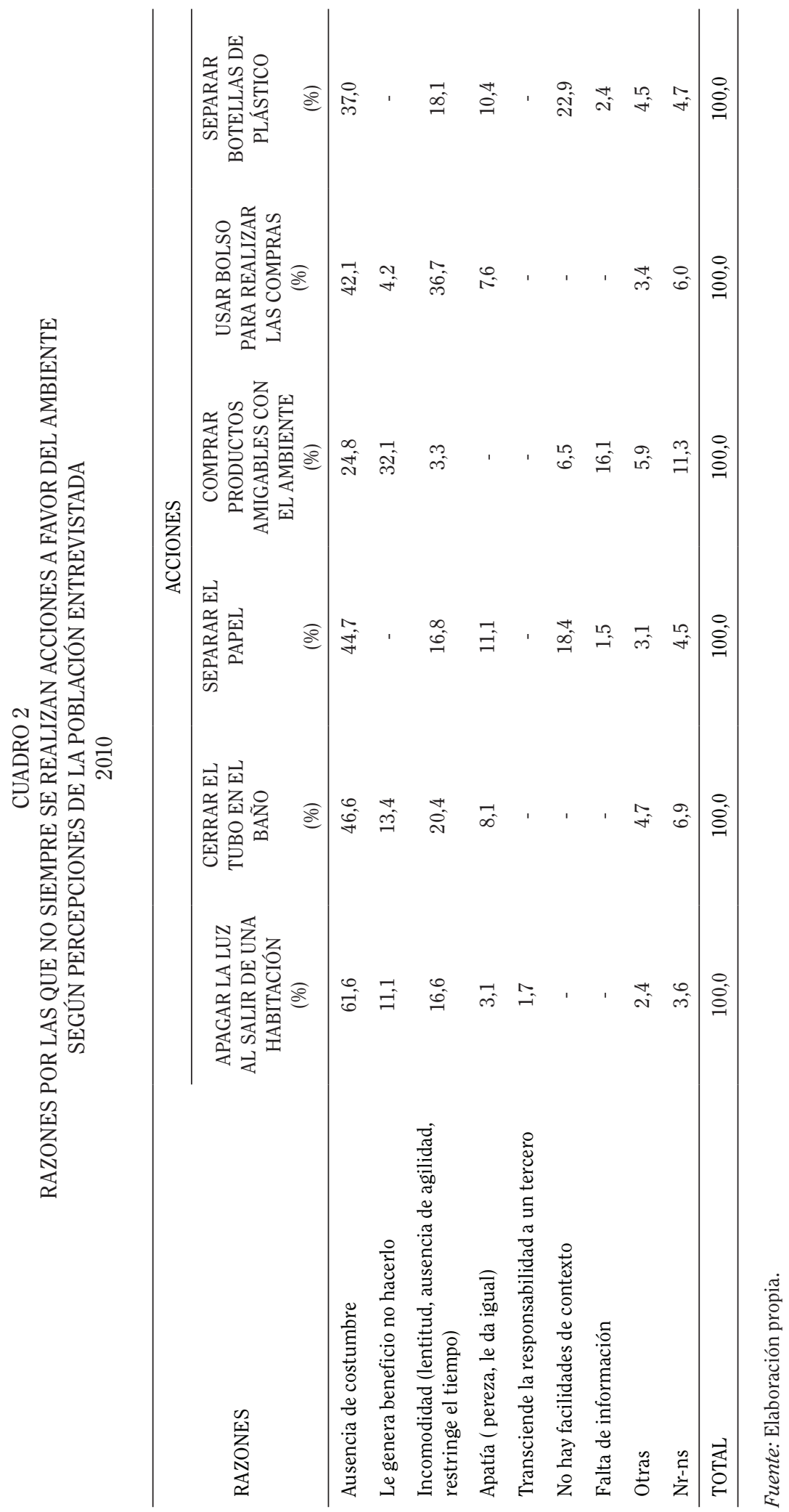


Se dieron respuestas donde se destaca un beneficio con no realizar la acción, por ejemplo, cuando se refiere a apagar las luces, se justifica no hacerlo por: seguridad del hogar o el gusto por la claridad; en el caso de cerrar el tubo, se justifica no hacerlo por la sensación de placer que produce estar debajo del agua. Este tipo de respuestas aparecen con gran frecuencia, cuando se preguntan las razones por las que no se escoge siempre los productos amigables con el ambiente, se tiende a mencionar la fidelidad hacia un producto determinado y los precios más bajos de otros productos.

Otros tipos de respuesta se enfocan en la incomodidad que genera realizar la acción. Hay connotación que la acción ambiental limita el tiempo o bien, genera algún otro tipo de incomodidad. Este tipo de respuesta presenta entre 15\% y $20 \%$ de mención en la mayoría de las acciones consultadas, con excepción de la compra de productos amigables con el ambiente, que casi no presentó menciones de incomodidad.

Es importante resaltar que esta categoría de respuesta presenta similitud con la descrita anteriormente, ambas se encuentran unidas por el factor confort que genera el no realizar la acción. La diferencia es que en la categoría anterior, se tiende a destacar la presencia de un beneficio cuando la acción no es realizada, mientras que en esta categoría hay una connotación de prejuicio con realizar la acción.

La categoría de apatía alude a la falta de interés de actuar proambientalmente. En las acciones de separación de desechos es donde hay mayor presencia de apatía, aunque se debe destacar que su mención no es amplia.

La ausencia de facilidad de contexto remite en el caso de separación de desechos, debido a la ausencia de lugares en donde dejar los desechos. Este tipo de justificación tuvo un peso considerable dentro de las respuestas brindadas en las acciones de separación de desechos. Por otro lado, la ausencia de facilidad de contexto en la acción de compra de productos amigables con el ambiente responde a la dificultad de encontrar los productos.

La falta de información obedece básicamente al desconocimiento sobre cómo llevar a cabo la separación de desechos o bien, al desconocimiento de que existen productos amigables con el ambiente.

$\mathrm{Al}$ analizar la relación entre las características sociodemográficas y el comportamiento proambiental en acciones cotidianas, se encuentra una relación significativa entre la edad y el puntaje total de la escala (ver cuadro 3 ).

CUADRO 3

RELACIÓN ENTRE CARACTERÍSTICAS

SOCIODEMOGRÁFICAS Y ESCALA DE

COMPORTAMIENTO PROAMBIENTAL

(COEFICIENTES DE UN MODELO

DE REGRESIÓN LINEAL MCO)

\begin{tabular}{lcc}
\hline \multirow{2}{*}{ VARIABLES } & \multicolumn{2}{c}{ ACCIONES } \\
\cline { 2 - 3 } & \multicolumn{2}{c}{ COTIDIANAS } \\
\hline Constante & 57.404 & .000 \\
Ruralidad & 0.0764 & .597 \\
Mujer vs. Hombre & 1.085 & .303 \\
Edad (incremento 10 años) & 2.3 & .000 \\
Secundaria o más vs. Primaria & -1.445 & .267 \\
Universidad vs. Primaria & -0.230 & .889 \\
Tenencia artefactos & -0.836 & .000 \\
\hline
\end{tabular}

Fuente: Elaboración propia.

Nota: B representa la magnitud del coeficiente y p permite determinar si el coeficiente es significativo al 5\% de significancia.

El modelo de MCO cumple con el supuesto de homocedasticidad. Incumple el supuesto de normalidad de residuos; no obstante, el tamaño de muestra es suficientemente grande como para que los estimadores MCO sigan una distribución normal asintóticamente.

Hay mayor tendencia de llevar a cabo acciones ambientales cotidianas en las personas de mayor edad, es decir, conforme aumenta la edad se da un aumento en la frecuencia con que las personas actúan proambientalmente.

También el nivel socioeconómico muestra influencia significativa sobre el comportamiento ambiental, ello se concluye a partir de la relación que hay entre el indicador de tenencia de artefactos del hogar y el puntaje de la escala (cuadro 1). Al incrementar el indicador de tenencia de artefactos del hogar, se disminuye el puntaje total de la escala. Ahora bien, es 
importante aclarar que la relación encontrada entre esas dos variables es débil. Al incrementar en una unidad el indicador de tenencia de artefactos (que varía de 0 a 10), se disminuye en un promedio de 0,8 unidades el puntaje de la escala (que varía de 0 a 100).

El compromiso ambiental en la realización de denuncias se evalúa de dos formas: una es preguntando a las personas que alguna vez se han enterado de que alguna empresa o persona esté contaminando o destruyendo la naturaleza sin ningún control, si ellas han puesto alguna denuncia al respecto. La otra es preguntado a las personas que han visto alguna fuga de agua en la calle, si ellas han puesto la denuncia cuando se dieron cuenta.
Cerca del $20 \%$ de las personas entrevistadas (238 personas) se han dado cuenta de alguien que está contaminando o destruyendo la naturaleza sin ningún control. De ese total, solo el 45\% afirma haber puesto la denuncia. Por su parte, $83 \%$ de las personas entrevistadas (997 personas) recuerda haber visto alguna vez una fuga de agua en la calle. De esa cantidad, el $44 \%$ las ha reportado cada vez que las ha visto.

Entre las razones por las que no se reportan las denuncias, figura la falta de credibilidad de que la denuncia se haga efectiva, esta respuesta fue la más mencionada en lo que respecta a denuncias por contaminación o destrucción de la naturaleza (24\% de las personas establecen esta razón) (ver cuadro 4).

CUADRO 4

RAZONES POR LAS QUE NO SE REALIZAN LAS DENUNCIAS O REPORTES SEGÚN PERCEPCIONES DE LA POBLACIÓN ENTREVISTADA 2010

\begin{tabular}{lcc}
\hline & CONTAMINACIÓN/ DESTRUCCIÓN NATURALEZA & \multicolumn{2}{c}{ FUGAS DE AGUA } \\
RAZONES & $(\%)$ & 13,5 \\
& 23,7 & 29,7 \\
Ineficiencia de la denuncia & 21,8 & 15,2 \\
Indiferencia a la situación & 19,9 & 19,2 \\
Desconocimiento a quien dirigirse & 10,2 & 12,2 \\
Otras personas denuncian & 3,1 & - \\
No contaba con el tiempo & 2,2 & 7,3 \\
Las personas contaminan & 9,9 & 2,9 \\
Otros & 9,2 & 100,0 \\
No sabe/No responde & 100,0 & \\
\hline TOTAL & & 2,0 \\
\hline
\end{tabular}

Fuente: Elaboración propia.

Hay gran indiferencia hacia las situaciones que ameritan denuncia o reporte, por ejemplo, en el caso de reporte de fugas de agua, la indiferencia se muestra como la principal razón para no realizarlo, respuestas como: "no es mi problema", "no es mi hogar", "no me corresponde", adicionan al $30 \%$ de quienes no realizaron la denuncia.

Se encuentra que hay una parte importante que desconoce dónde y cómo realizar la denuncia, $y$ otra parte, que no realiza la denuncia bajo el argumento de que otro ya la hizo. Otras respuestas que presentan menor mención remiten al tiempo como justificante para no denunciar o bien, al argumento de que todas o la mayoría de las personas contaminan y por ende, no denuncian.

El compromiso hacia la participación en campañas ambientales se indaga preguntando a las personas si en los últimos dos años, han 
participado en alguna campaña en beneficio de la naturaleza, como: campañas de recolección de basura, siembra de árboles, limpieza de ríos, playas, etc. Apenas un $35 \%$ de las personas entrevistadas responden afirmativamente.

A quienes no participan, se pregunta por las razones de no hacerlo. Nuevamente, aparece el factor tiempo como justificante para no actuar de forma proambiental y en este caso resulta ser la respuesta más brindada (29\% de las personas que no participan aluden a esta razón) (ver cuadro 5).

CUADRO 5

RAZONES POR LAS QUE NO SE PARTICIPA EN

CAMPAÑAS DE BENEFICIO A LA NATURALEZA

SEGÚN PERCEPCIONES DE LA POBLACIÓN

ENTREVISTADA 2010

\begin{tabular}{lc}
\hline RAZONES & $\%$ \\
\hline Falta de tiempo/está muy ocupado & 29,4 \\
$\begin{array}{l}\text { No ha escuchado/no sabe donde hacerlo/no se } \\
\text { ha presentado la oportunidad }\end{array}$ & 25,8 \\
$\begin{array}{l}\text { Donde vive no se hace nada/se realiza lejos de } \\
\text { donde vive }\end{array}$ & 21,5 \\
Le da pereza/no le interesa/no le gusta & 9,3 \\
Problemas de salud & 4,6 \\
Por la edad & 2,6 \\
Otras & 3,1 \\
Nr/ns & 3,7 \\
\hline TOTAL & 100,0 \\
\hline
\end{tabular}

Fuente: Elaboración propia.

La falta de información se presenta en segunda instancia, como justificante para no participar en campañas en beneficio del ambiente. En tercera instancia, se presenta un factor de contexto como lo es la ausencia de actividades en el lugar de residencia. Otras respuestas establecen el desinterés hacia realizar la acción o bien, los problemas físicos como justificantes.

Para analizar la relación entre las características sociodemográficas y el compromiso con realizar denuncias o participar en campañas ambientales, se corren modelos de regresión logística. Los resultados de los modelos se observan en el cuadro 6.

La edad influye significativamente en el compromiso con realizar denuncias por fuga de agua, independientemente del sexo, nivel educativo, ruralidad del distrito de residencia y nivel socioeconómico del hogar. Conforme aumenta la edad, aumentan las posibilidades de que una persona denuncie un acto de destrucción o contaminación de la naturaleza. En lo que se refiere a denuncias por daño a la naturaleza, la edad no ejerce influencia significativa.

Además, existe relación entre el urbanismo del distrito de residencia y el compromiso con reportar fugas de agua. A mayor urbanismo, menor es la posibilidad de que las personas reporten las fugas de agua.

El sexo presenta relación significativa con la participación en campañas de beneficio ambiental. Las mujeres tienden a participar menos que los hombres, independientemente de la edad, escolaridad, ruralidad del distrito de residencia y nivel socioeconómico del hogar. La edad mostró influencia significativa sobre la participación en actividades. Se encuentra que a mayor edad, menores son las posibilidades de participación. El nivel educativo también ejerce influencia sobre la participación, se observa que las personas con universidad completa tienden a participar más que las personas con primaria completa o menos. 
CUADRO 6

RELACIÓN ENTRE CARACTERÍSTICAS SOCIODEMOGRÁFICAS,

EL COMPROMISO CON LA PARTICIPACIÓN EN CAMPAÑAS Y LA REALIZACIÓN DE DENUNCIAS

(COEFICIENTES DE LOS MODELOS DE REGRESIÓN LOGÍSTICA)

\begin{tabular}{lcccccc}
\hline \multirow{2}{*}{ VARIABLES } & \multicolumn{2}{c}{$\begin{array}{c}\text { PARTICIPACIÓN EN } \\
\text { ACTIVIDADES }\end{array}$} & \multicolumn{2}{c}{$\begin{array}{c}\text { DENUNCIA POR } \\
\text { CONTAMINACIÓN }\end{array}$} & \multicolumn{2}{c}{$\begin{array}{c}\text { DENUNCIA POR } \\
\text { FUGAS DE AGUA }\end{array}$} \\
\cline { 2 - 7 } & $\mathrm{B}$ & $\mathrm{p}$ & $\mathrm{B}$ & $\mathrm{p}$ & $\mathrm{B}$ & $\mathrm{P}$ \\
\hline Urbanismo & .99 & .44 & .93 & .08 & .91 & .00 \\
Mujer vs. hombre & .65 & .00 & 1.45 & .22 & 1.07 & .64 \\
Edad (incremento 10 años) & .91 & .02 & .84 & .10 & 1.40 & .00 \\
Secundaria o más vs. primaria & 1.02 & .90 & 1.57 & .25 & .78 & .17 \\
Universidad vs. primaria & 1.96 & .00 & .76 & .53 & .84 & .42 \\
Tenencia artefactos & 1.02 & .33 & 1.01 & .89 & .96 & .15 \\
\hline
\end{tabular}

Fuente: Elaboración propia.

Nota: B representa la magnitud del coeficiente y p permite determinar si el coeficiente es significativo al $5 \%$ de significancia.

\section{DISCUSIÓN}

Como es de esperarse, la población no asume las distintas acciones ambientales cotidianas con la misma frecuencia y compromiso, por ejemplo, las acciones de ahorro de energía y agua son realizadas por la gran mayoría de la población, mientras que las acciones de separación de desechos y hábitos de compra proambientales son frecuentadas por cerca de la mitad de la población o menos.

Resulta interesante el hecho de que las acciones que generan un ahorro monetario directo a los hogares, son las que precisamente se llevan más a cabo. Si el impulso para realizar esas acciones ambientales está más promovido por el beneficio económico que por un impulso de conservación y protección del ambiente, cabe preguntarse ¿qué tanta conciencia ambiental tiene la población? No obstante, esta pregunta solo es parte de una conjetura que valdría la pena retomar en estudios posteriores.

$\mathrm{Al}$ evaluar la escala de acciones ambientales cotidianas se obtiene un puntaje de 64 , lo cual indica que en términos globales, la población presenta cierta tendencia de acción proambiental, aunque no se puede considerar alta ni predominantemente. También es importante rescatar que tan solo un tercio de la población parece mostrar una conducta predominantemente proambiental. Aunque estos resultados no son comparables con los analizados por Lentini (2005), hay coincidencia en que la mayor tendencia de la población es hacia una conducta parcialmente responsable.

Esta escala de acciones ambientales cotidianas permitirá evaluar de forma global los cambios en la conducta ambiental cotidiana de la población a través del tiempo, pero es necesario crear una escala individualizada para los diferentes tipos de estas acciones, con la finalidad de tomar el pulso a las diferentes conductas ambientales.

Con respecto a las razones que brindan las personas entrevistadas para no actuar siempre de forma proambiental en acciones cotidianas, se hallan varios factores; sin embargo, son dos los más determinantes: uno responde a la ausencia de costumbre (olvidar o no recordar realizar siempre la acción ambiental) y el otro, a un asunto de confort (realizar la acción genera algún inconveniente). Ambos factores podrían estar asociados a muchas cosas como la falta de preocupación por el ambiente, bajo sentido de responsabilidad ambiental o ausencia de conciencia ambiental. 
Un factor importante que parece inhibir la conducta de separación de desechos, es el contexto o el entorno, cerca de una quinta parte de las personas que no separan los desechos argumentan la ausencia de lugares para llevar los desechos separados como motivo para no realizar la acción.

Menos de la mitad de las personas que son testigos de una situación que afecta el ambiente actúa siempre de forma responsable. La desconfianza hacia la efectividad de la denuncia se presenta como un factor importante que inhibe el accionar para poner la denuncia, otro factor es el desconocimiento hacia dónde acudir. Ambos factores están relacionados con las circunstancias del entorno que pueden promover o inhibir el comportamiento ambiental (Stern, 2000: 421).

La mayoría de las personas no han participado en campañas de beneficio ambiental en los últimos dos años. Un elemento que parece tener influencia sobre esta situación es la desinformación sobre dónde y cuándo se realizan las campañas.

La indiferencia hacia la realización de las acciones ambientales está presente en diferentes niveles, en algunas acciones se presenta más acrecentada que en otras. Esa indiferencia parece tener su origen en actitudes individualistas.

En todas las acciones ambientales consultadas, pero sobre todo en la de participación en campañas ambientales, hay respuestas que aluden al tiempo como razón para no actuar de forma proambiental. Quizá el propio sistema en el que vivimos, el cual presiona el tiempo en función de la producción y adquisición, es un elemento inhibidor de la conducta ambiental.

Son muchos los estudios que intentan explicar la conducta ambiental desde diferentes enfoques y teorías, pero resulta difícil encontrar alguno que evalúe de forma directa la influencia del propio modelo económico, la dinámica de vida de las personas y el propio individualismo sobre la conducta ambiental. A partir de los resultados hallados en esta investigación, se sugiere abordar los elementos anteriores en futuros estudios que intenten explicar la conducta ambiental de la población costarricense.
Al analizar la relación entre las variables sociodemográficas y la conducta ambiental, se llega a varias conclusiones. Una es que la edad ejerce influencia significativa sobre la conducta ambiental cotidiana y sobre la denuncia de situaciones que destruyen y contaminan el ambiente. Las personas más jóvenes tienden a actuar en menor medida proambientalmente, lo cual es coherente con lo hallado en otras investigaciones en países latinos que han monitoreado la conducta de ahorro de agua (Pato y Tamayo, 2006: 59; González, 2002: 144).

También se halla que a mayor urbanismo, menor tendencia a reportar las fugas de agua, esto podría tener alguna relación con las dinámicas de vida de las regiones, considerando que en zona urbana el tiempo se restringe aún más. Por último, se observa que las mujeres participan menos que los hombres en campañas de beneficio ambiental. No solo en actividades ambientales las mujeres presentan menor participación, esta situación también se replica en otros tipos de participación social (Carillo et ál., 2001).

\section{BIBLIOGRAFÍA}

\section{LIBROS}

Corcoran, P.; Vilela, M. y Roerink, A. La carta de la tierra en acción. Amsterdam: KIT Publishers, 2005.

Corral, Víctor. Estudios de psicología ambiental en América Latina. México: UAP-UNAMCONACyT, 1998.

Gudynas, Eduardo. Ecología, economía y ética del desarrollo de América Latina. Montevideo: Coscoroba, 2002.

Hassan, P. La carta de la tierra en acción. Amsterdam: KIT Publishers, 2005.

Mackey, B. La carta de la tierra en acción. Amsterdam: KIT Publishers, 2005.

Tucker, E. La carta de la tierra en acción. Amsterdam: KIT Publishers, 2005.

\section{REVISTAS}

Barr, Stewart y Gilg, Andrew. "A conceptual framework for understanding and analyzing attitudes towards 
environmental behavior". Journal Compilation 89 (4). 2007: 361-379.

Berger, Ida. "The demographics of recycling and the structure of environmental behavior". Environment and Behavior 29 (4). 1997: 515-531.

Bustos Vásquez, A.; Padilla, R.; Pernudi, V.; Sandoval, I. y Solórzano, N. "Percepción de la población costarricense sobre el ambiente". Serie Pulso Nacional 39. Heredia, Universidad Nacional, 2005: $1-14$.

Bustos Vásquez, A.; Pernudi, V.; Sandoval, I.; Segura, G. y Solórzano, N. "Percepción de la población costarricense hacia el ambiente". Serie Pulso Nacional 46. Heredia, Universidad Nacional, 2006: $1-22$.

Carillo, María et ál. "La población costarricense del Gran Área Metropolitana frente a su percepción sobre la democracia, participación ciudadana y el proceso electoral". Serie Pulso Nacional 16. Heredia. Universidad Nacional, 2001: 2-44. Datos inéditos.

Granzin, Kent y Olsen, Janeen. "Characterizing participants in activities protecting the environment: a focus on donating, recycling and conservation behaviors". Journal of Public Policy and Marketing 10 (2).American Marketing Association, 1991: 1-27.

Hines, Jody; Hungerford, Harold y Tomera, Audrey. "Analysis and synthesis of research on responsible environmental behavior: a meta-analysis". The Journal of Environmental Education 18 (2). 1986: 1-8.

Lentini, Valeria. "Valores ambientales de ticos y panameños". Ambientico 136. Escuela de
Ciencias Ambientales de la Universidad Nacional de Costa Rica, 2005: 16-21.

Pato, Claudia y Tamayo, Álvaro. "Valores, creencias ambientales y comportamiento ecológico de activismo". Medio ambiente y comportamiento humano 7 (1). 2006: 51-66.

Sandoval, Irma et ál. "La población costarricense del Gran Área Metropolitana frente a su percepción sobre la democracia, participación ciudadana y el proceso electoral". Serie Pulso Nacional 12. Heredia. Universidad Nacional, 2001: 2-44.

Stern, Paul. "Toward a coherent theory of environmentally significant behavior". Journal of Social Issues 56 (3). 2000: 407-424.

TESIS

González, Antonio. "La preocupación por la calidad del medio ambiente. Un modelo cognitivo sobre la conducta ecológica”. [Tesis Doctoral en Psicología]. Universidad Complutense de Madrid, 2002

Sandoval, Irma; Pernudi, Vilma y Carrillo, María. "Percepción de la población costarricense sobre el ambiente". Presentación. 2007. [En línea] En: <www.una.ac.cr/idespo/index. php?option $=$ com_booklibrary\&task $=v$ iew\&id $=62 \&$ catid $=49 \&$ Itemid $=96>$. Consultado el 5 abril de 2010.

Fecha de ingreso: 05/04/2011 Fecha de aprobación: 18/01/2012 